\title{
Effect of Using Tanniniferous Plants Shrubs in Feeding Barki Ewes on some Productive and Physiological Parameters
}

EL-Gohary, E. S. H. ${ }^{1}$; M. M. Fathala ${ }^{3}$; M. A-H. El-Rayes $^{2}$; R. M. Abou-Aiana ${ }^{1}$ and M. M. Eissa ${ }^{1}$

${ }^{1}$ Animal Production Research Institute, Agriculture Research Center, Ministry of Agriculture, Doki, Giza, Eygpt.

${ }^{2}$ Animal and Poultry Physiology Department, Desert Research Center, Matareya, Cairo, Egypt.

${ }^{3}$ Animal Husbandry and Animal wealth Development, Faculty of Veterinary Medicine Alexandria University, Egypt.

Corresponding author: e-mails: EMAD SALAH EL-GOHARY, dr_emad_goh@yahoo.com or emadelgohary739@yahoo.com

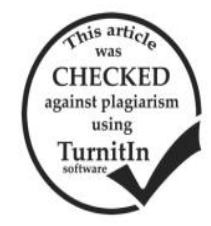

\begin{abstract}
Tanniniferous plants shrubs are those plants which tolerate the high levels of salts in the soil, they are well grown in the northern coast of Egypt, and they include Acacia nilotica, Atriplex nummularia and Cassava manihot esculenta. This study aimed at exploring the possibility of complete replacement of berseem hay (control) in the diet by leaves and stems of tanniniferous shrubs plants and its effect on productive and physiological responses, in addition to some milk and blood biochemical constituents. Forty mature healthy Barki ewes at late pregnancy, 3-4 years old with an average live body weight (LBW) $51.5 \pm 0.46 \mathrm{~kg}$ were randomly divided into 4 similar groups. The $1^{\text {st }}$ group (Hay) was fed the control diet which consisted of $40 \%$ concentrate feed mixture plus $60 \%$ berseem hay $(\mathrm{BH})$, while in the $2^{\text {nd }}, 3^{\text {rd }}$ and $4^{\text {th }}$ groups, $\mathrm{BH}$ as percentage was replaced by Acacia, Atriplex and Cassava respectively. Body weight of ewes and their offsprings were recorded biweekly. Milk yield was measured weekly and milk samples of each animal were taken for milk analysis. Blood samples were collected biweekly for measuring some hematological and biochemical parameters. Feeding tanniniferous plants shrubs did not result in significant differences on body weight of Barki ewes and their offsprings, while improved milk productivity and increased milk protein and lactose. According to the results of biochemical parameters of blood, it could be concluded that feeding tanniniferous plants shrubs did not cause any negative effects on blood parameters of the experimental animals under the present study.
\end{abstract}

Keywords: Barki ewes, tanniniferous plants, milk, blood parameters.

\section{INTRODUCTION}

Sheep play an important role in agriculture environment especially in desert lands. Feed stuffs and fresh water scarcity are the main restraining factors for adequate animal production under the arid conditions in Egypt. Sheep, goats and camels always over graze the palatable plants. The less and inedible plant species represent roughly $70 \%$ of the total green coverage (El-Shaer et al., 1997). Thus under these challenging conditions, unusual feed resources such as tanniniferous plants shrubs and other salt-tolerant plants can offer practical alternatives in marginal areas as stated by ElShaer and Gihad (1994) and Squires and Ayoub (1994). The edible parts of these plants could be used as animal feed resources, mainly in dry seasons.

Tanniniferous plants shrubs under study include Acacia nilotica, Atriplex nummularia and Cassava manihot esculenta. Mixing tanniniferous plants shrubs into rations is recommended for feeding animals. Tanniniferous plants shrubs are distinguished with minimum use of brackish or saline irrigating water and tolerable to arid or semi-arid conditions. They also give good biomass yield which could be used for feeding sheep and goat (ELSaadany et al., 2016).

Atriplex is characterized by moderate digestible crude protein and high oxalate, while digestible ether extract and soluble carbohydrates are low (El-Shaer and Gihad, (1994) and Ben Salem et al. (2002). Moreover, due to high ash content, Atriplex foliage is relatively low in energy contents. It is reported that sheep fed on Atriplex only decreased or maintained their live weights (Hassan and Abdel-Aziz, 1979 and Warren et al., 1990). In the presence of energy sources such as barely grains, Atriplex proved to be an excellent source of alternative nitrogen supplement for sheep that fed low quality roughage as cereal straws (Ben Salem et al.,
2005). Goats can depend unharmed in their feeding on Atriplex halimus and Acacia saligna plus barley (100\% of their energy maintenance requirements) without any serious influence for less than 26 weeks (Ibrahim, 2001).

Feeding of tanniniferous plants shrubs is considered a suitable solution to conquer the expected lack of feed and its scarcity in Egypt (Shawket et al., 2010). This forage shrubs species are well grown in the northern coast of Egypt (Degan, et al., 1997). Feeding tanniniferous plants shrubs for a long duration exhibited changes in the body fluids, haemogram and histopathology of different organs, which did not return back to normal conditions after exclusion of the plants from the diet (Ibrahim, 2001).

Barki sheep lambs' LBW or average daily gain (ADG) did not affected by grazing on more than one type of forages shrubs like Atriplex halimus and Acacia saligna under arid and semi-arid conditions when compared with feeding berseem hay without unfavorable biological effects (Mohammady et al., 2014).

Milk yield and milk composition of ruminants vary by system of feeding, breed, parity, season of feeding, managerial practices, environmental circumstances, lactation stage and health state of the Mammary gland. Sheep milk contains high total solids and major nutrient contents. Many changes in sheep milk composition occurred during the late stage of lactation because towards the end of lactation the fat, protein, total solids and minerals contents increased, while the lactose content decreased (Haenlein, 2001).

Furthermore, Vongsamphanh and Wanapat (2004) found that feeding of high levels of Cassava hay and dried Cassava root increased the milk yield and decreased the feed conversion ratio and feed cost, while concentration of blood urea-N were not affected by the level of Cassava hay in the diet. 
Somatic cell count (SCC) is a measure of the white blood cell figure in milk. The level of SCC in milk of a particular ewe reflects the health condition of her mammary glands. Recently, researchers have indicated that the upper threshold for SCC in udder of healthy ewes should be 250,000 cells/ml (Pengov, 2001 and Menzies and Ramanoon, 2001).

Therefore, this study aimed at exploring the possibility of complete replacement of berseem hay (control) in the diet by leaves and stems of tanniniferous shrubs plants (Acacia nilotica, Atriplex nummularia and Cassava manihot esculenta) and its effect on some productive and physiological responses, in addition to some milk and blood biochemical constituents.

\section{MATERIALS AND METHODS}

This study was carried out at Borg El-Arab Experimental station, Alexandria Governorate, belonging to the Animal Production Research Institute, Agricultural Research Center, Ministry of Agriculture, whereas the fodder trees of Acacia niloitca, Atriplex nummularia and Cassava manihot esculenta were harvested along the subroads of the North Western Coast of Egypt near the Mediterranean Sea, west of Alexandria city, latitudes $21^{\circ}$ and $31^{\circ}$ North and longitudes $25^{\circ}$ and $35^{\circ}$ East.

\section{Animals and experimental managements:}

Forty mature healthy Barki ewes at late pregnancy, aged 3-4 years with average live body weight $51.5 \pm 0.46 \mathrm{~kg}$ were used in this study. The animals were randomly divided into 4 similar groups (10 ewes each) according to their live body weight (LBW) and age. All animals were kept in a semi-open shaded yard and kept under the same managerial conditions during the experimental periods.

Animals of the first group (G1) were fed the control diet consisted of $40 \%$ concentrate feed mixture (CFM), plus $60 \%$ berseem hay $(\mathrm{BH})$, while in the $2^{\text {nd }}, 3^{\text {rd }}$ and $4^{\text {th }}$ groups, $\mathrm{BH}$ as percentage had been replaced by leaves and stems of Acacia, Atriplex and Cassava, respectively. The CFM consisted of $25 \%$ un decorticated cotton meal, $43 \%$ yellow corn, $25 \%$ wheat bran, $3.5 \%$ molasses, $2 \%$ limestone, $1 \%$ common salt and $0.5 \%$ minerals mixtures.

Animals were fed on experimental diets to cover their nutrient allowances according to the physiological and productive stage (NRC, 1985). Ewes were adapted to experimental diets for 4 weeks as preliminary period, and then fed experimental rations 4 weeks before parturition and continued to weaning their lambs. All animals were fed daily at 9 a.m. and 4 p.m., fresh water and block minerals were available all times. Chemical composition on DM\% (DM, OM, CP, EE, NFE and Ash), of feed stuffs were analyzed according to A.O.A.C. (1995) Table (1).

Table 1. Chemical composition of feed ingredients on DM\% basis

\begin{tabular}{lccccc}
\hline \multirow{2}{*}{ Item } & \multicolumn{5}{c}{ Experimental Feed Stuff } \\
\cline { 2 - 6 } & CFM & Hay & Acacia & Atriplex & Cassava \\
\hline & Chemical & Composition & on & DM\% basis & \\
DM & 91.20 & 95.12 & 71.62 & 45.32 & 44.39 \\
OM & 93.09 & 89.59 & 81.88 & 73.92 & 88.26 \\
CP & 15.70 & 10.64 & 10.03 & 12.19 & 22.94 \\
CF & 14.23 & 38.54 & 16.54 & 25.12 & 28.05 \\
EE & 3.13 & 1.03 & 1.74 & 1.72 & 2.92 \\
NFE & 60.84 & 39.38 & 53.57 & 34.89 & 34.35 \\
Ash & 6.10 & 10.41 & 18.12 & 26.08 & 11.74 \\
\hline
\end{tabular}

Experimental measurements and samples collection:

Experimental periods were consisted of 2 intervals, late pregnancy (4 weeks before lambing) and suckling period ( 8 weeks). Live body weights (LBW) of ewes and their lambs were recorded biweekly. Milk yield was measured weekly throughout the lactation period by using of milk suckling technique in which, lambs were isolated from their mothers during previous night and body weight was recorded (to the nearest $10 \mathrm{gm}$ ) at the morning (7 a.m.). Lambs were left to suckle their dams for 30 minutes, and then immediately were weighed again. The difference between pre and post-suckling weights was defined as milk consumption or milk intake. The residual milk was hand milked and recorded. Similar procedures were repeated at the evening suckling at 5.30 p.m. difference between pre and post-suckling weights were added to previous milk intake quantity to calculate the daily milk intake (milk consumption) of suckling lambs. Milk intake plus milk removed by hand milking represented daily milk yield. All lambs were weaned irrespective of weight at 8 weeks of age. Individual milk samples of each ewe in each group, representing morning and evening milking were collected weekly from the $2^{\text {nd }}$ up to $7^{\text {th }}$ wk. of the suckling period. Approximately $100 \mathrm{ml}$ milk of each animal was sampled for the determination of milk composition. Somatic cell count (SCC) was determined using milk analyzer, (model EKOMILK-M, Eon Trading LLC, USA). The SCC was converted into the somatic cell score (SCS) according to the following equation that given by Wiggans and Shook (1987):-

$$
\mathrm{SCS}=\log 2[\mathrm{SCC} /(\mathbf{1 0 0 , 0 0 0})+3]
$$

Milk samples were directly analyzed for fat, total solids (TS) and total proteins using the methods described by Ling (1963). Lactose content was determined calorimetrically according to Barnett and Abd El-Tawab (1957). Ash content was determined according the methods reported in A.O.A.C. (1984).

Blood samples were collected biweekly during experimental periods from the jugular vein of ewes into clean test tubes with anticoagulant. Blood samples were divided into two portions. In the $1^{\text {st }}$ portion, hematological parameters including count of red (RBC's $\left.\times 10^{6} / \mathrm{mm}^{3}\right)$ and white (WBC's $\times 10^{3} / \mathrm{mm}^{3}$ ) blood cells, hematocrit value (Ht $\%$ ) and hemoglobin ( $\mathrm{Hg} \mathrm{g} / \mathrm{dl})$ concentration in the whole blood were immediately measured after collection. The $2^{\text {nd }}$ portion was centrifuged at $3000 \mathrm{rpm}$ for 20 minutes to obtain plasma and frozen at $-20 \stackrel{\mathrm{C}}{\mathrm{C}}$ for late biochemical assay. Plasma concentrations total proteins, albumin, total 
Lipids, triglycerides, total cholesterol, total antioxidant capacity (TAC), glucose, liver activity enzymes and kidney functions were estimated calorimetrically using commercial chemical reagent kits (Bio-diagnostic product Kit, Egypt). However, globulin concentration and albumin/globulin ratio were calculated.

\section{Statistical analysis:}

Live body weights of ewes and their offspring, average weekly milk yield, milk composition were statistically analyzed using General Linear Model's procedures of SAS GLM (SAS, 2004), the model includes the effect of treatments (four variables). Means were tested using (Duncan's multiple Range test procedure 1995).

Blood hematological and biochemical parameters were statistically analyzed by General Linear Model's procedures of SAS GLM (SAS, 2004). The model includes the effect of treatments (four variables), sampling time (three times) and their interaction. Means were compared via the LSMEANS/PDIFF of the same procedure. Values were considered significant at $\mathrm{P} \leq 0.05$.

\section{RESULTS AND DISCUSSION}

\section{Impact of feeding tanniniferous plants shrubs on: Live body weight (LBW) of ewes:}

Results show the influence of feeding tanniniferous plants shrubs (Atriplex, Acacia and Cassava) as alternatives to $\mathrm{BH}$ on live body weights (LBW) of Barki ewes (Figure 1). There was no significant differences between animals in the initial (LBW) at the start of experiment (4 weeks pre-lambing). Moreover, at lambing and at 4 weeks post-lambing LBW of Barki ewes revealed also insignificant differences between the different experimental groups. At weaning ( 8 weeks post-lambing) there was significant difference between ewes fed Acacia and those fed Hay (50.90 vs. 47.40 kg.). Animals fed Acacia recorded the highest LBW when compared with the other experimental groups.

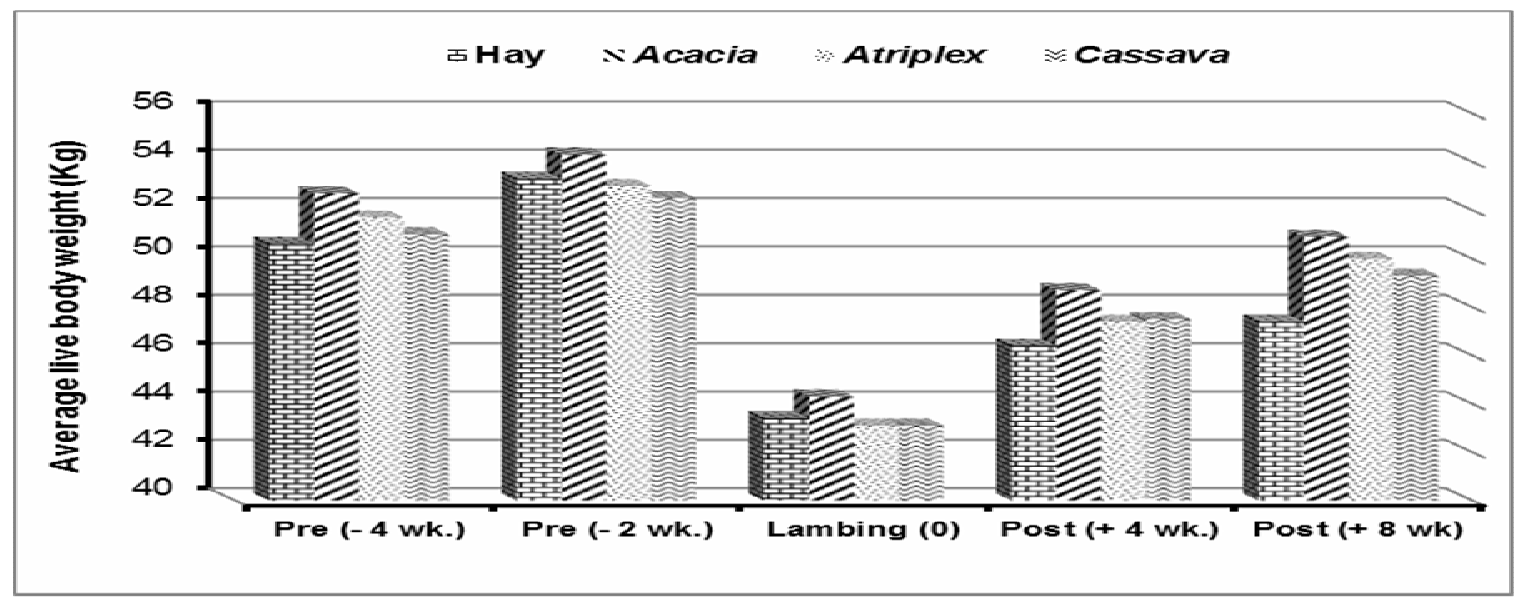

Figure 1. Changes of live body weights $(\mathrm{kg})$ of Barki ewes pre and post lambing.

The present results are in the same direction with those obtained by EL-Saadany et al. (2016) who recorded insignificant differences among different experimental groups (Acacia, Atriplex and Cassava vs. berseem hay) in LBW of Barki ewes during the 15 days pre-partum, at lambing and at 60 days post-partum. Likewise, AbuZanat, and Tabbaa (2006) and Shaker et al. (2014) found insignificant differences due to adding Atriplex and Acacia in ration of ewes and Shami goats. These obtained results revealed that these forage shrubs can be used for maintaining growth and body weight of sheep as berseem hay $(\mathrm{BH})$ without negative impacts, although these plants have high ash and low energy contents especially Acacia and Cassava.

\section{Average weekly milk production:}

Statistical results presented in Table (2) show the effect of feeding tanniniferous plants shrubs on milk yield of Barki ewes under the experiment. Generally Barki ewes fed Cassava produced the highest $(\mathrm{P}<0.05)$ total milk yield followed by ewes fed Hay, and then ewes fed Atriplex and Acacia, respectively. The milk yield of ewes in different experimental groups increased till the end of $3^{\text {rd }}$ week then decreased gradually till the end of lactation period. The peaks of average weekly milk yield that recorded in the $3^{\text {rd }}$ week from lambing were 10.783, $8.483,6.441$ and $10.018 \mathrm{~kg}$ for Cassava, Atriplex,
Acacia, and Hay groups respectively. Concerning the average daily milk yield $\mathrm{kg} / \mathrm{head}$, the highest $(\mathrm{P}<0.05)$ value was recorded by ewes fed Cassava $(1.052 \mathrm{~kg} / \mathrm{head})$, while the averages of other groups were $0.662,0.814$ and $0.916 \mathrm{~kg} / \mathrm{head}$ for ewes fed Acacia, Atriplex and Hay respectively.

In agreement with the present results, Abu-Zanat and Tabbaa (2006) found that the highest milk yield was recorded by ewes fed Cassava when compared to those fed other types of forages. They attributed the high milk yield due to higher dietary $\mathrm{CP}$ and $\mathrm{EE}$ in Cassava. In contrast, Shawket et al. (2010) found that inclusion of fresh Atriplex instead of $\mathrm{BH}$ in the diet increased $(\mathrm{P}<0.05)$ the milk production of camel.

\section{Live body weight of newly born lambs:}

Table (2) demonstrates the results of LBW of newly born lambs from lambing to the $8^{\text {th }}$ week of age after birth. Feeding tanniniferous plants shrubs (Acacia, Atriplex and Cassava as alternatives to berseem hay) did not exhibit any significant differences between the different experimental groups. There were no significant differences in lambs birth weight between experimental groups. The highest values of biweekly live body weights, total body gain and average daily gain of newly born lambs were recorded in animals fed Cassava while the lowest recorded in those fed Acacia. 
The concurrent results come in agreement with those obtained by Shetaewi et al. (2001); Fasae et al. (2015) and EL-Saadany et al. (2016) who recorded differences $(\mathrm{P}<0.05)$ between ewes fed Acacia, Atriplex and Cassava when compared with those fed Berseem hay in total gain and average daily gain of lambs. On the other hand, these results are not in accordance with those obtained by Mohammady et al. (2014) who reported higher weaning weight and daily gain $(\mathrm{P}<0.05)$ in Barki lambs fed $\mathrm{BH}$ when compared with those fed tanniniferous plants shrubs.

Table 2. Impact of feeding tanniniferous plants shrubs on average weekly milk yield of Barki ewes and live body weight changes of newly born lambs. (Means $\pm \mathrm{SE}$ )

\begin{tabular}{|c|c|c|c|c|}
\hline \multirow{2}{*}{ Lactation period $(\mathrm{Kg} / \mathrm{h})$} & \multicolumn{4}{|c|}{ Experimental group } \\
\hline & Hay & Acacia & Atriplex & Cassava \\
\hline \multicolumn{5}{|c|}{ Weekly milk yield(kg/h) } \\
\hline $1^{\text {st }} w k$. & $5.231 \pm 0.32^{\mathrm{a}}$ & $4.080 \pm 0.26^{\mathrm{b}}$ & $4.795 \pm 0.31^{\mathrm{ab}}$ & $5.111 \pm 0.32^{\mathrm{a}}$ \\
\hline $2^{\text {nd }} w k$. & $7.926 \pm 0.65^{\mathrm{a}}$ & $4.788 \pm 0.16^{\mathrm{b}}$ & $6.150 \pm 0.48^{\mathrm{b}}$ & $8.855 \pm 0.51^{\mathrm{a}}$ \\
\hline $3^{\text {rd }} \mathrm{wk}$. & $10.018 \pm 0.29^{\mathrm{a}}$ & $6.441 \pm 0.35^{\mathrm{c}}$ & $8.483 \pm 0.72^{b}$ & $10.783 \pm 0.54^{\mathrm{a}}$ \\
\hline $4^{\text {th }} \mathrm{wk}$. & $7.576 \pm 0.30^{b}$ & $5.824 \pm 0.08^{\mathrm{c}}$ & $6.955 \pm 0.48^{\mathrm{b}}$ & $9.431 \pm 0.24^{\mathrm{a}}$ \\
\hline $5^{\text {th }} \mathrm{wk}$. & $6.389 \pm 0.34^{\mathrm{b}}$ & $4.929 \pm 0.26^{\mathrm{c}}$ & $5.975 \pm 0.47 b^{c}$ & $7.936 \pm 0.46^{\mathrm{a}}$ \\
\hline $6^{\text {th }} w k$. & $5.978 \pm 0.32^{\mathrm{b}}$ & $4.303 \pm 0.22^{\mathrm{c}}$ & $5.003 \pm 0.26^{\mathrm{c}}$ & $6.955 \pm 0.48^{\mathrm{a}}$ \\
\hline $7^{\text {th }}$ wk. & $4.466 \pm 0.43^{\mathrm{bc}}$ & $3.573 \pm 0.20^{\mathrm{c}}$ & $4.536 \pm 0.24^{\mathrm{b}}$ & $5.531 \pm 0.33^{\mathrm{a}}$ \\
\hline $8^{\text {th }} w k$. & $3.685 \pm 0.30^{\mathrm{ab}}$ & $3.111 \pm 0.13^{\mathrm{b}}$ & $3.679 \pm 0.36^{\mathrm{ab}}$ & $4.324 \pm 0.49^{\mathrm{a}}$ \\
\hline Total milk yield/kg/h & $51.269 \pm 1.76^{\mathrm{b}}$ & $37.048 \pm 1.41^{\mathrm{d}}$ & $45.575 \pm 2.58^{\mathrm{c}}$ & $58.926 \pm 1.86^{\mathrm{a}}$ \\
\hline Average daily milk yield $(\mathrm{kg} / \mathrm{h} / \mathrm{d})$ & $0.916 \pm 0.03^{\mathrm{b}}$ & $0.662 \pm 0.03^{\mathrm{d}}$ & $0.814 \pm 0.05^{\mathrm{c}}$ & $1.052 \pm 0.03^{\mathrm{a}}$ \\
\hline \multicolumn{5}{|c|}{ Changes in live body weights $(\mathrm{kg})$ of lambs } \\
\hline Lamb's birth weight & $3.678 \pm 0.10^{\mathrm{a}}$ & $3.700 \pm 0.14^{\mathrm{a}}$ & $3.789 \pm 0.17^{\mathrm{a}}$ & $3.676 \pm 0.24^{\mathrm{a}}$ \\
\hline After 2 & $7.027 \pm 0.15^{\mathrm{a}}$ & $6.615 \pm 0.52^{\mathrm{a}}$ & $6.868 \pm 0.31^{\mathrm{a}}$ & $7.448 \pm 0.39^{\mathrm{a}}$ \\
\hline After 4 weeks & $10.367 \pm 0.23^{\mathrm{a}}$ & $9.530 \pm 0.96^{\mathrm{a}}$ & $9.946 \pm 0.63^{\mathrm{a}}$ & $11.020 \pm 0.58^{\mathrm{a}}$ \\
\hline After 6 weeks & $11.136 \pm 0.22^{\mathrm{a}}$ & $10.610 \pm 0.95^{\mathrm{a}}$ & $11.231 \pm 0.52^{\mathrm{a}}$ & $11.871 \pm 0.48^{\mathrm{a}}$ \\
\hline After 8 weeks & $11.818 \pm 0.34^{\mathrm{a}}$ & $11.370 \pm 0.96^{\mathrm{a}}$ & $11.975 \pm 0.47^{\mathrm{a}}$ & $12.520 \pm 0.75^{\mathrm{a}}$ \\
\hline Total gain & $8.140 \pm 0.31^{\mathrm{a}}$ & $7.670 \pm 0.92^{\mathrm{a}}$ & $8.186 \pm 0.55^{\mathrm{a}}$ & $8.844 \pm 0.70^{\mathrm{a}}$ \\
\hline Average daily gain & $0.145 \pm 0.01^{\mathrm{a}}$ & $0.137 \pm 0.02^{\mathrm{a}}$ & $0.146 \pm 0.01^{\mathrm{a}}$ & $0.158 \pm 0.01^{\mathrm{a}}$ \\
\hline
\end{tabular}

a, $b$ and $c$, Means within the same row with different superscript are significantly differ $(\mathbf{P}<0.05)$.

\section{Chemical composition of milk:}

Data in Table (3) revealed that there were no significant differences between the different experimental groups for overall means of milk fat $\%$ that were $6.56,6.70$, 6.86 and $6.78 \%$ for Hay, Acacia, Atriplex and Cassava groups, respectively. However, ewes fed Acacia showed the highest $(\mathrm{P}<0.05)$ milk fat $(7.90 \%)$ at the $6^{\text {th }}$ week of lactation. It worth notice, that these ewes also gave the least $(\mathrm{P}<0.05)$ weekly yield during the same week.

Ewes of Hay group had the lowest $(\mathrm{P}<0.05)$ milk protein\% $(3.77 \%)$. Milk protein percentages in other groups were 3.95, 3.99 and 3.93\% for Acacia, Atriplex and Cassava, respectively.

The same trend of protein $\%$ was also occurred for lactose $\%$, and the overall means were 4.82, 5.06, 5.13 and $5.05 \%$ for Hay, Acacia, Atriplex and Cassava groups, respectively.

Ewes of Hay group showed the lowest $(\mathrm{P}<0.05)$ overall mean of milk ash $(0.79 \%)$, while those fed Acacia had the highest $(0.95 \%)$. Overall means of milk total solids percentages revealed insignificant differences and were 17.18, 17.88, 18.01 and 17.61\% for Hay, Acacia, Atriplex and Cassava groups, respectively.

With reference to somatic cell score (SCC) there was no difference between experimental groups. Overall means of SCC were 4.503, 4.691, 4.193 and 4.502 for Acacia, Atriplex and Cassava groups, respectively.

The concurrent results match those obtained by Shawket and Ibrahem (2012) who reported that substitution of Atriplex for berseem hay in the diet caused marked increase $(\mathrm{P}<0.05)$ in protein content of camel milk.
However, Ahmed et al. (2013) found no effect to feeding tanniniferous plants shrubs on milk constituents. Moreover, Shetaewi et al. (2001) reported that milk fat percentages decreased in does fed concentrate diet plus green Acacia in comparison to those fed concentrate diet plus BH. They attributed this result to the higher digestibility coefficients of the nutrients in berseem hay than those in Acacia leaves. Generally, in the present study feeding lactating ewes with tanniniferous plants shrubs as alternative to berseem hay resulted in increasing milk protein, lactose and ash percentages, while did not affect fat and total solids percentages and somatic cell score. Based on these results we can say that using of tanniniferous plants shrubs as alternative to berseem hay did not show adverse effects on mammary glands of experimental Barki ewes.

\section{Ewes blood biochemical:}

\section{A. Blood picture:}

Feeding tanniniferous plants shrubs revealed an increase $(\mathrm{P}<0.05)$ in red blood corpuscles $(\mathrm{RBC}$ 's), white blood corpuscles (WBC's), hemoglobin ( $\mathrm{Hb})$ and hematocrit \% (Ht \%) than found in ewes fed $\mathrm{BH}$, as shown in Table (4). The highest values of these parameters were exhibited by ewes fed Cassava plant.

For the effect of the physiological status, ewes fed $\mathrm{BH}$ did not show big variation from pre- to post-partum, while those fed tanniniferous plants showed significant decrease in all these parameters after parturition.

These results come in opposite direction to those results obtained by Abdelhameed et al. (2006); ElBassiony (2013) and El-Hawy (2013) who reported 
highly significant decrease in $R B C$ 's and WBC's in different animals species (sheep, goats, camels, and cattle) that fed tanniniferous plants when compared to

the control group which fed BH. In addition, Abdelhameed et al. (2006) found insignificant effect to feeding tanniniferous plants shrubs on $\mathrm{Hb}$ and $\mathrm{Ht} \%$.

Table 3. Milk composition of Barki ewes fed tanniniferous plants shrubs during suckling period. (Means $\pm \mathrm{SE}$ )

\begin{tabular}{|c|c|c|c|c|c|}
\hline \multirow{2}{*}{ Item } & \multirow{2}{*}{ Suckling Wk. } & \multicolumn{4}{|c|}{ Experimental group } \\
\hline & & Hay & Acacia & Atriplex & Cassava \\
\hline \multirow{7}{*}{ Fat $\%$} & $2^{\text {nd }} w k$ & $6.47 \pm 0.442^{\mathrm{a}}$ & $6.85 \pm 0.340^{\mathrm{a}}$ & $6.80 \pm 0.218^{\mathrm{a}}$ & $6.83 \pm 0.300^{\mathrm{a}}$ \\
\hline & $3^{\text {rd }} \mathrm{wk}$ & $6.93 \pm 0.365^{\mathrm{a}}$ & $6.16 \pm 0.485^{\mathrm{a}}$ & $7.40 \pm 0.301^{\mathrm{a}}$ & $7.15 \pm 0.420^{\mathrm{a}}$ \\
\hline & $4^{\text {th }}$ wk. & $5.70 \pm 0.650^{\mathrm{a}}$ & $6.15 \pm 0.344^{\mathrm{a}}$ & $6.73 \pm 0.301^{\mathrm{a}}$ & $6.54 \pm 0.370^{\mathrm{a}}$ \\
\hline & $5^{\text {th }}$ wk. & $6.60 \pm 0.287^{\mathrm{a}}$ & $6.28 \pm 0.548^{\mathrm{a}}$ & $6.93 \pm 0.433^{\mathrm{a}}$ & $6.95 \pm 0.321^{\mathrm{a}}$ \\
\hline & $6^{\text {th }}$ wk. & $6.51 \pm 0.537^{\mathrm{b}}$ & $7.90 \pm 0.207^{\mathrm{a}}$ & $6.61 \pm 0.370^{\mathrm{b}}$ & $6.71 \pm 0.225^{\mathrm{b}}$ \\
\hline & $7^{\text {th }}$ wk. & $6.91 \pm 0.300^{\mathrm{a}}$ & $6.64 \pm 0.444^{\mathrm{a}}$ & $6.58 \pm 0.335^{\mathrm{a}}$ & $6.49 \pm 0.331^{\mathrm{a}}$ \\
\hline & Overall mean $\pm \mathrm{SE}$ & $6.56 \pm 0.254^{\mathrm{a}}$ & $6.70 \pm 0.220^{\mathrm{a}}$ & $6.86 \pm 0.224^{\mathrm{a}}$ & $6.78 \pm 0.157^{\mathrm{a}}$ \\
\hline \multirow{7}{*}{ Protein \% } & $2^{\text {nd }}$ wk. & $3.81 \pm 0.061^{\mathrm{b}}$ & $4.00 \pm 0.066^{\mathrm{a}}$ & $4.09 \pm 0.069^{\mathrm{a}}$ & $3.94 \pm 0.038^{\mathrm{ab}}$ \\
\hline & $3^{\text {rd }} \mathrm{wk}$ & $3.81 \pm 0.061^{\mathrm{b}}$ & $3.88 \pm 0.059^{\mathrm{b}}$ & $4.05 \pm 0.050^{\mathrm{a}}$ & $3.98 \pm 0.049^{\mathrm{ab}}$ \\
\hline & $4^{\text {th }}$ wk. & $3.66 \pm 0.124^{b}$ & $3.95 \pm 0.071^{\mathrm{a}}$ & $3.99 \pm 0.035^{\mathrm{a}}$ & $3.99 \pm 0.044^{\mathrm{a}}$ \\
\hline & $5^{\text {th }}$ wk. & $3.89 \pm 0.023^{\mathrm{a}}$ & $3.94 \pm 0.068^{\mathrm{a}}$ & $3.89 \pm 0.035^{\mathrm{a}}$ & $3.85 \pm 0.153^{\mathrm{a}}$ \\
\hline & $6^{\text {th }}$ wk. & $3.59 \pm 0.160^{\mathrm{b}}$ & $3.90 \pm 0.057^{\mathrm{a}}$ & $3.99 \pm 0.055^{\mathrm{a}}$ & $3.86 \pm 0.063^{\mathrm{a}}$ \\
\hline & $7^{\text {th }}$ wk. & $3.88 \pm 0.045^{\mathrm{b}}$ & $4.06 \pm 0.053^{\mathrm{a}}$ & $3.95 \pm 0.057^{\mathrm{ab}}$ & $3.98 \pm 0.073^{\mathrm{ab}}$ \\
\hline & Overall mean $\pm \mathrm{SE}$ & $3.77 \pm 0.033^{\mathrm{b}}$ & $3.95 \pm 0.041^{\mathrm{a}}$ & $3.99 \pm 0.035^{\mathrm{a}}$ & $3.93 \pm 0.041^{\mathrm{a}}$ \\
\hline \multirow{7}{*}{ Lactose \% } & $2^{\text {nd }}$ wk. & $4.88 \pm 0.075^{b}$ & $5.10 \pm 0.097^{\mathrm{ab}}$ & $5.29 \pm 0.103^{\mathrm{a}}$ & $5.06 \pm 0.068^{\mathrm{ab}}$ \\
\hline & $3^{\text {rd }} \mathrm{wk}$ & $4.85 \pm 0.085^{\mathrm{b}}$ & $4.94 \pm 0.080^{\mathrm{b}}$ & $5.21 \pm 0.086^{\mathrm{a}}$ & $5.09 \pm 0.088^{\mathrm{ab}}$ \\
\hline & $4^{\text {th }}$ wk. & $4.88 \pm 0.086^{\mathrm{b}}$ & $5.04 \pm 0.091^{\mathrm{ab}}$ & $5.09 \pm 0.052^{\mathrm{ab}}$ & $5.13 \pm 0.053^{\mathrm{a}}$ \\
\hline & $5^{\text {th }} \mathrm{wk}$. & $4.94 \pm 0.038^{\mathrm{a}}$ & $5.05 \pm 0.107^{\mathrm{a}}$ & $4.96 \pm 0.050^{\mathrm{a}}$ & $4.93 \pm 0.229^{\mathrm{a}}$ \\
\hline & $6^{\text {th }} \mathrm{wk}$ & $4.40 \pm 0.257^{\mathrm{b}}$ & $4.99 \pm 0.074^{\mathrm{a}}$ & $5.14 \pm 0.091^{\mathrm{a}}$ & $4.94 \pm 0.093^{\mathrm{a}}$ \\
\hline & $7^{\text {th }} w k$. & $4.98 \pm 0.060^{\mathrm{a}}$ & $5.24 \pm 0.087^{\mathrm{a}}$ & $5.06 \pm 0.091^{\mathrm{a}}$ & $5.13 \pm 0.113^{\mathrm{a}}$ \\
\hline & Overall mean $\pm \mathrm{SE}$ & $4.82 \pm 0.063^{\mathrm{b}}$ & $5.06 \pm 0.060^{\mathrm{a}}$ & $5.13 \pm 0.056^{\mathrm{a}}$ & $5.05 \pm 0.062^{\mathrm{a}}$ \\
\hline \multirow{7}{*}{ Ash \% } & $2^{\text {nd }} w k$ & $0.76 \pm 0.060^{\mathrm{a}}$ & $0.92 \pm 0.103^{\mathrm{a}}$ & $0.89 \pm 0.081^{\mathrm{a}}$ & $0.94 \pm 0.076^{\mathrm{a}}$ \\
\hline & $3^{\text {rd }} \mathrm{wk}$ & $0.78 \pm 0.056^{\mathrm{a}}$ & $1.00 \pm 0.124^{\mathrm{a}}$ & $0.93 \pm 0.116^{\mathrm{a}}$ & $0.89 \pm 0.106^{\mathrm{a}}$ \\
\hline & $4^{\text {th }}$ wk. & $0.69 \pm 0.040^{\mathrm{a}}$ & $0.86 \pm 0.073^{\mathrm{a}}$ & $0.84 \pm 0.107^{\mathrm{a}}$ & $0.86 \pm 0.080^{\mathrm{a}}$ \\
\hline & $5^{\text {th }}$ wk. & $0.78 \pm 0.073^{\mathrm{a}}$ & $0.79 \pm 0.105^{\mathrm{a}}$ & $0.75 \pm 0.050^{\mathrm{a}}$ & $0.68 \pm 0.041^{\mathrm{a}}$ \\
\hline & $6^{\text {th }}$ wk. & $0.85 \pm 0.107^{b}$ & $1.21 \pm 0.111^{\mathrm{a}}$ & $0.98 \pm 0.127^{\mathrm{ab}}$ & $0.84 \pm 0.114^{\mathrm{b}}$ \\
\hline & $7^{\text {th }}$ wk. & $0.88 \pm 0.094^{\mathrm{a}}$ & $0.93 \pm 0.127^{\mathrm{a}}$ & $0.94 \pm 0.098^{\mathrm{a}}$ & $0.86 \pm 0.068^{\mathrm{a}}$ \\
\hline & Overall mean $\pm \mathrm{SE}$ & $0.79 \pm 0.022^{\mathrm{b}}$ & $0.95 \pm 0.041^{\mathrm{a}}$ & $0.89 \pm 0.047^{\mathrm{ab}}$ & $0.84 \pm 0.042^{\mathrm{ab}}$ \\
\hline \multirow{7}{*}{ Total solids \% } & $2^{\text {nd }}$ wk. & $17.60 \pm 0.806^{\mathrm{a}}$ & $18.06 \pm 0.427^{\mathrm{a}}$ & $18.06 \pm 0.374^{\mathrm{a}}$ & $17.81 \pm 0.301^{\mathrm{a}}$ \\
\hline & $3^{\text {rd }}$ wk. & $17.36 \pm 0.453^{b}$ & $16.98 \pm 0.511^{\mathrm{b}}$ & $19.23 \pm 0.714^{\mathrm{a}}$ & $18.10 \pm 0.468^{\mathrm{ab}}$ \\
\hline & $4^{\text {th }}$ wk. & $15.93 \pm 0.692^{\mathrm{a}}$ & $17.29 \pm 0.605^{\mathrm{a}}$ & $17.64 \pm 0.377^{\mathrm{a}}$ & $17.51 \pm 0.455^{\mathrm{a}}$ \\
\hline & $5^{\text {th }}$ wk. & $17.20 \pm 0.302^{\mathrm{a}}$ & $17.29 \pm 0.904^{\mathrm{a}}$ & $17.86 \pm 0.627^{\mathrm{a}}$ & $17.40 \pm 0.618^{\mathrm{a}}$ \\
\hline & $6^{\text {th }}$ wk. & $16.35 \pm 0.795^{\mathrm{b}}$ & $19.33 \pm 0.400^{\mathrm{a}}$ & $17.71 \pm 0.515^{\mathrm{b}}$ & $17.35 \pm 0.193^{b}$ \\
\hline & $7^{\text {th }}$ wk. & $18.63 \pm 0.795^{\mathrm{a}}$ & $18.34 \pm 0.898^{\mathrm{a}}$ & $17.53 \pm 0.407^{\mathrm{a}}$ & $17.45 \pm 0.478^{\mathrm{a}}$ \\
\hline & Overall mean $\pm \mathrm{SE}$ & $17.18 \pm 0.352^{\mathrm{a}}$ & $17.88 \pm 0.362^{\mathrm{a}}$ & $18.01 \pm 0.258^{\mathrm{a}}$ & $17.61 \pm 0.151^{\mathrm{a}}$ \\
\hline \multirow{7}{*}{ Somatic cell score } & $2^{\text {nd }}$ wk. & $4.361 \pm 0.378^{\mathrm{a}}$ & $4.778 \pm 0.375^{\mathrm{a}}$ & $4.260 \pm 0.237^{\mathrm{a}}$ & $5.060 \pm 0.435^{\mathrm{a}}$ \\
\hline & $3^{\text {rd }}$ wk. & $3.447 \pm 0.203^{\mathrm{a}}$ & $3.808 \pm 0.380^{\mathrm{a}}$ & $3.715 \pm 0.300^{\mathrm{a}}$ & $3.912 \pm 0.460^{\mathrm{a}}$ \\
\hline & $4^{\text {th }} \mathrm{wk}$. & $4.700 \pm 0.684^{\mathrm{a}}$ & $3.776 \pm 0.266^{\mathrm{ab}}$ & $3.263 \pm 0.169^{b}$ & $3.545 \pm 0.273^{\mathrm{ab}}$ \\
\hline & $5^{\text {th }}$ wk. & $3.247 \pm 0.147^{\mathrm{a}}$ & $4.020 \pm 0.504^{\mathrm{a}}$ & $3.891 \pm 0.240^{\mathrm{a}}$ & $3.830 \pm 0.457^{\mathrm{a}}$ \\
\hline & $6^{\text {th }} \mathrm{wk}$ & $5.149 \pm 0.231^{\mathrm{a}}$ & $5.866 \pm 0.310^{\mathrm{a}}$ & $5.182 \pm 0.334^{\mathrm{a}}$ & $5.038 \pm 0.256^{\mathrm{a}}$ \\
\hline & $7^{\text {th }}$ wk. & $3.065 \pm 0.040^{\mathrm{a}}$ & $3.288 \pm 0.225^{\mathrm{a}}$ & $2.987 \pm 0.013^{\mathrm{a}}$ & $3.058 \pm 0.074^{\mathrm{a}}$ \\
\hline & Overall mean $\pm \mathrm{SE}$ & $4.503 \pm 0.227^{\mathrm{a}}$ & $4.691 \pm 0.264^{\mathrm{a}}$ & $4.193 \pm 0.180^{\mathrm{a}}$ & $4.502 \pm 0.260^{\mathrm{a}}$ \\
\hline
\end{tabular}

a and $b$, Means within the same row with different superscript are significantly differ $(\mathrm{P}<0.05)$.

\section{B. Protein fractions:}

Blood plasma total proteins (TP) and its fractions, albumin (Al) and globulin (Gl) have a great importance as good indicators of nutritional status. Feeding tanniniferous plants shrubs resulted in significant increase in plasma concentration of total proteins and albumin as shown in Table (5). The highest mean of total proteins was recorded by ewes fed Cassava, while the highest mean of albumin was recorded by ewes fed Acacia. Plasma globulin did not affected by feeding tanniniferous plants. As a result albumin to globulin ratio $(\mathrm{Al} / \mathrm{Gl})$ appreciably increased in plasma of ewes fed these plants. In general plasma protein increased by feeding tanniniferous plants through increasing albumin fraction. Albumin is known to play an important role in body fluids regulation hence coping with salt stress (Abdel-Bary, 1990).

The effects of physiological status were not constant on plasma protein fractions. Total plasma proteins increased in all groups from pre- to post-partum reaching the highest values at the end of lactation period. For plasma albumin it increased in ewes fed $\mathrm{BH}$, but decreased in ewes fed Cassava plant. In the other two groups, albumin decreased after parturition then increased again at time of weaning lambs. Plasma globulin while increased in ewes fed $\mathrm{BH}$ it decreased in those fed tanniniferous plants. 
EL-Saadany et al. (2016) found significant increase in total plasma protein concentration between experimental groups (Acacia, Atriplex and Cassava vs. Hay). With regard to albumin, Shaker et al. (2008) recorded that plasma albumin was higher in Barki lambs fed mixture of Acacia and Atriplex. For globulin concentrations, the obtained results come in accordance with those obtained by El-hawy (2013) who recorded insignificant difference in doe goats by feeding mixture of Atriplex and Acacia compared to BH feeding.

However, the present results differ from those obtained by Badawy et al. (2002) and Shaker et al. (2008 and 2014) who reported that feeding fresh Acacia decreased plasma TP, Al and Gl values in growing Barki lambs. Similarly, Ibrahim (2001) reported that goats fed Atriplex or Acacia had lower value of TP compared with those fed BH. In addition, Badawy et al. (2002) reported that the animals fed Atriplex nummularia or Acacia saligna showed lower Al/Gl ratio.

In general, the present results showed clearly that all values of plasma protein fractions were within the normal reference ranges of these metabolites. This indicated that the animals under the study were in good nutritional status and their liver had no damage since these metabolites are mainly synthesized in it.

Table 4. Means \pm SE of some blood parameters of Barki ewes fed tanniniferous plants shrubs during different physiological periods.

\begin{tabular}{|c|c|c|c|c|c|}
\hline \multirow{2}{*}{ Item } & \multirow{2}{*}{ Sampling time } & \multicolumn{4}{|c|}{ Experimental group } \\
\hline & & Hay & Acacia & Atriplex & Cassava \\
\hline \multirow{4}{*}{$\mathrm{RBC}$ 's $\left(10^{6} / \mathrm{mm}^{3}\right)$} & Pre-parturition & $4.852 \pm 0.151^{\mathrm{d}}$ & $6.398 \pm 0.174^{b}$ & $5.775 \pm 0.284^{\mathrm{c}}$ & $7.394 \pm 0.306^{\mathrm{a}}$ \\
\hline & At lambing & $4.939 \pm 0.129^{\mathrm{a}}$ & $4.748 \pm 0.0593^{\mathrm{a}}$ & $4.900 \pm 0.019^{\mathrm{a}}$ & $5.079 \pm 0.099^{\mathrm{a}}$ \\
\hline & At weaning & $4.610 \pm 0.206^{\mathrm{ab}}$ & $4.570 \pm 0.045^{\mathrm{b}}$ & $4.676 \pm 0.072^{\mathrm{ab}}$ & $5.051 \pm 0.078^{\mathrm{a}}$ \\
\hline & Overall mean $\pm \mathrm{SE}$ & $4.799 \pm 0.096^{\mathrm{c}}$ & $5.239 \pm 0.164^{\mathrm{b}}$ & $5.117 \pm 0.129 b^{c}$ & $5.841 \pm 0.23^{\mathrm{a}}$ \\
\hline \multirow{4}{*}{ WBC's $\left(10^{3} / \mathrm{mm}^{3}\right)$} & Pre-parturition & $9.988 \pm 0.308^{\mathrm{c}}$ & $11.292 \pm 0.697^{\mathrm{b}}$ & $11.885 \pm 0.585^{b}$ & $15.214 \pm 0.631^{\mathrm{a}}$ \\
\hline & At lambing & $10.163 \pm 0.265^{\mathrm{a}}$ & $9.891 \pm 0.083^{\mathrm{a}}$ & $9.986 \pm 0.115^{\mathrm{a}}$ & $10.435 \pm 0.206^{\mathrm{a}}$ \\
\hline & At weaning & $9.486 \pm 0.425^{\mathrm{a}}$ & $9.405 \pm 0.093^{\mathrm{a}}$ & $9.622 \pm 0.149^{\mathrm{a}}$ & $10.394 \pm 0.162^{\mathrm{a}}$ \\
\hline & Overall mean $\pm \mathrm{SE}$ & $9.879 \pm 0.196^{\mathrm{b}}$ & $10.196 \pm 0.272^{\mathrm{b}}$ & $10.498 \pm 0.270^{\mathrm{b}}$ & $12.010 \pm 0.47^{\mathrm{a}}$ \\
\hline \multirow{4}{*}{$\mathrm{Hg}(\mathrm{g} / \mathrm{dl})$} & Pre-parturition & $9.460 \pm 0.291^{\mathrm{c}}$ & $12.428 \pm 0.336^{b}$ & $12.255 \pm 0.493^{b}$ & $14.408 \pm 0.5970^{\mathrm{a}}$ \\
\hline & At lambing & $9.669 \pm 0.234^{\mathrm{a}}$ & $9.359 \pm 0.114^{\mathrm{a}}$ & $9.447 \pm 0.116^{\mathrm{a}}$ & $10.023 \pm 0.210^{\mathrm{a}}$ \\
\hline & At weaning & $8.983 \pm 0.403^{b}$ & $8.895 \pm 0.086^{\mathrm{b}}$ & $9.102 \pm 0.143^{\mathrm{ab}}$ & $9.846 \pm 0.154^{\mathrm{a}}$ \\
\hline & Overall mean $\pm \mathrm{SE}$ & $9.371 \pm 0.184^{\mathrm{c}}$ & $10.227 \pm 0.314^{\mathrm{b}}$ & $10.268 \pm 0.312^{\mathrm{b}}$ & $11.430 \pm 0.44^{\mathrm{a}}$ \\
\hline \multirow{4}{*}{ Ht (\%) } & Pre-parturition & $31.218 \pm 0.962^{\mathrm{d}}$ & $41.143 \pm 1.120^{b}$ & $37.135 \pm 1.829^{c}$ & $47.548 \pm 1.969^{\mathrm{a}}$ \\
\hline & At lambing & $31.900 \pm 0.775^{\mathrm{a}}$ & $30.730 \pm 0.310^{\mathrm{a}}$ & $31.580 \pm 0.125^{\mathrm{a}}$ & $32.810 \pm 0.614^{\mathrm{a}}$ \\
\hline & At weaning & $29.643 \pm 1.328^{\mathrm{ab}}$ & $29.390 \pm 0.290^{\mathrm{b}}$ & $30.070 \pm 0.465^{\mathrm{ab}}$ & $32.482 \pm 0.507^{\mathrm{a}}$ \\
\hline & Overall mean $\pm \mathrm{SE}$ & $30.920 \pm 0.608^{c}$ & $33.754 \pm 1.048^{\mathrm{b}}$ & $32.928 \pm 0.829^{\mathrm{bc}}$ & $37.610 \pm 1.47^{\mathrm{a}}$ \\
\hline
\end{tabular}

a, b, c and d, Means within the same row with different superscript are significantly differ $(\mathbf{P}<0.05)$.

Table 5. Effect of feeding tanniniferous plants shrubs on protein fractions of Barki ewes during different physiological periods. (Means $\pm \mathrm{SE}$ )

\begin{tabular}{|c|c|c|c|c|c|}
\hline \multirow{2}{*}{ Item } & \multirow{2}{*}{ Sampling time } & \multicolumn{4}{|c|}{ Experimental group } \\
\hline & & Hay & Acacia & Atriplex & Cassava \\
\hline \multirow{4}{*}{ Total Protein, g/dl } & Pre-parturition & $6.268 \pm 0.263^{c}$ & $7.120 \pm 0.142^{b}$ & $7.189 \pm 0.114^{\mathrm{ab}}$ & $7.781 \pm 0.414^{\mathrm{a}}$ \\
\hline & At lambing & $7.662 \pm 0.220^{\mathrm{ab}}$ & $7.874 \pm 0.115^{\mathrm{a}}$ & $7.674 \pm 0.261^{\mathrm{ab}}$ & $7.129 \pm 0.131^{b}$ \\
\hline & At weaning & $8.141 \pm 0.220^{\mathrm{a}}$ & $8.168 \pm 0.221^{\mathrm{a}}$ & $8.058 \pm 0.157^{\mathrm{a}}$ & $8.587 \pm 0.182^{a}$ \\
\hline & Overall mean $\pm \mathrm{SE}$ & $7.357 \pm 0197^{\mathrm{b}}$ & $7.721 \pm 0.123^{\mathrm{ab}}$ & $7.640 \pm 0.124^{\mathrm{ab}}$ & $7.832 \pm 0.187^{\mathrm{a}}$ \\
\hline \multirow{4}{*}{ Albumin, g/dl } & Pre-parturition & $3.728 \pm 0.200^{c}$ & $5.021 \pm 0.133^{b}$ & $4.951 \pm 0.128^{b}$ & $5.731 \pm 0.406^{\mathrm{a}}$ \\
\hline & At lambing & $4.417 \pm 0.321^{\mathrm{a}}$ & $4.871 \pm 0.145^{\mathrm{a}}$ & $4.621 \pm 0.365^{\mathrm{a}}$ & $4.366 \pm 0.299^{\mathrm{a}}$ \\
\hline & At weaning & $5.478 \pm 0.212^{\mathrm{ab}}$ & $5.471 \pm 0.137^{\mathrm{ab}}$ & $5.580 \pm 0.102^{\mathrm{a}}$ & $4.899 \pm 0.203^{b}$ \\
\hline & Overall mean $\pm \mathrm{SE}$ & $4.541 \pm 0.193^{\mathrm{b}}$ & $5.121 \pm 0.090^{\mathrm{a}}$ & $5.051 \pm 0.148^{\mathrm{a}}$ & $4.999 \pm 0.203^{\mathrm{a}}$ \\
\hline \multirow{4}{*}{ Globulin, g/dl } & Pre-parturition & $2.541 \pm 0.183^{\mathrm{a}}$ & $2.100 \pm 0.055^{b}$ & $2.238 \pm 0.074^{\mathrm{ab}}$ & $2.049 \pm 0.166^{b}$ \\
\hline & At lambing & $3.126 \pm 0.116^{\mathrm{a}}$ & $3.145 \pm 0.071^{\mathrm{a}}$ & $3.181 \pm 0.144^{\mathrm{a}}$ & $2.899 \pm 0.196^{\mathrm{a}}$ \\
\hline & At weaning & $2.662 \pm 0.191^{\mathrm{b}}$ & $2.697 \pm 0.143^{b}$ & $2.478 \pm 0.167^{\mathrm{b}}$ & $3.689 \pm 0.110^{\mathrm{a}}$ \\
\hline & Overall mean $\pm \mathrm{SE}$ & $2.777 \pm 0.104^{\mathrm{a}}$ & $2.645 \pm 0.096^{\mathrm{a}}$ & $2.632 \pm 0.105^{\mathrm{a}}$ & $2.879 \pm 0.153^{\mathrm{a}}$ \\
\hline \multirow{4}{*}{$\mathrm{Al} / \mathrm{Gl}$ ratio } & Pre-parturition & $1.529 \pm 0.136^{\mathrm{c}}$ & $2.407 \pm 0.092^{b}$ & $2.241 \pm 0.112^{b}$ & $2.966 \pm 0.315^{\mathrm{a}}$ \\
\hline & At lambing & $1.515 \pm 0.140^{\mathrm{a}}$ & $1.563 \pm 0.075^{\mathrm{a}}$ & $1.495 \pm 0.151^{\mathrm{a}}$ & $1.760 \pm 0.163^{\mathrm{a}}$ \\
\hline & At weaning & $2.182 \pm 0.206^{\mathrm{a}}$ & $2.081 \pm 0.126^{\mathrm{a}}$ & $2.337 \pm 0.152^{\mathrm{a}}$ & $1.344 \pm 0.077^{b}$ \\
\hline & Overall mean $\pm \mathrm{SE}$ & $1.742 \pm 0.108^{\mathrm{a}}$ & $2.017 \pm 0.085^{\mathrm{a}}$ & $2.024 \pm 0.104^{\mathrm{a}}$ & $2.023 \pm 0.173^{\mathrm{a}}$ \\
\hline
\end{tabular}

a, $b$ and $c$, Means within the same row with different superscript are significantly differ $(\mathrm{P}<0.05)$.

\section{Energy components:}

Feeding tanniniferous plants reduced insignificantly plasma total lipids while increased significantly the levels of triglycerides. Total cholesterol increased in plasma of ewes fed tanniniferous plants but only significantly in those fed Cassava when compared with ewes fed BH (Table 6).
Physiological status did not affect plasma total lipids. In spite of that, both triglycerides and total cholesterol increased from pre- to post-partum stage in all experimental groups. 
Plasma glucose increased significantly by feeding tanniniferous plants and from pre- to post-partum stage. This was more obvious in ewes fed Atriplex.

El-Bassiony (2013) and Shaker et al. (2014) worked on Shami goats and Barki sheep fed on sun-dried mixture of Prosopis, Acacia and Leucaena in comparison with those fed $\mathrm{BH}$, found a decrease in plasma total lipids and total cholesterol, while plasma glucose did not affected. Also Ibrahim (2001) and Shaker (2014) recorded a decrease in energy components concentration due to feeding goats tanniniferous plants, and they attributed this decrease to high content of tannins and salts. Hassan et al. (2015) recorded a decrease in goats plasma energy components due to feeding some forage shrubs. They attributed this decrease due to lower production of Total volatile fatty acids (VFA's) in rumen which are probably due to lower solubility of nitrogen and reduced availability of amino acids for production of VFA's.

\section{Liver and kidney functions:}

Feeding tanniniferous plants did not affect the concentration of both alanine amino transferase (ALT) and alkaline phosphatase (ALK-P). Concentration of aspartate aminotransferase (AST) increased significantly $(\mathrm{P}<0.05)$ by feeding Acacia and Atriplex (Table 7).

For the effect of physiological status, it was found that both AST and ALT in all experimental groups were at the highest concentration in plasma of ewes at pre-parturition stage indicating higher function of liver at late pregnancy. The level of these two enzymes decreased to the lowest level at the end of lactation period (at weaning lambs) that meaning alleviation of physiological stress on liver at this time.

Table 6. Concentrations of energy components in blood plasma of Barki ewes fed tanniniferous plants shrubs in different physiological status. (Means $\pm \mathrm{SE}$ )

\begin{tabular}{|c|c|c|c|c|c|}
\hline \multirow{2}{*}{ Items } & \multirow{2}{*}{$\begin{array}{l}\text { Sampling } \\
\text { Time }\end{array}$} & \multicolumn{4}{|c|}{ Experimental group } \\
\hline & & Hay & Acacia & Atriplex & Cassava \\
\hline \multicolumn{6}{|c|}{ Lipid fractions } \\
\hline \multirow{4}{*}{ Total Lipids, g/dl } & Pre-parturition & $8.08 \pm 0.169^{\mathrm{a}}$ & $8.36 \pm 0.505^{\mathrm{a}}$ & $7.73 \pm 0.341^{\mathrm{a}}$ & $7.79 \pm 0.343^{\mathrm{a}}$ \\
\hline & At lambing & $7.17 \pm 0.541^{\mathrm{a}}$ & $7.45 \pm 0.138^{\mathrm{a}}$ & $6.62 \pm 0.447^{\mathrm{a}}$ & $6.82 \pm 0.419^{\mathrm{a}}$ \\
\hline & At weaning & $8.18 \pm 0.217^{\mathrm{a}}$ & $7.49 \pm 0.225^{b}$ & $8.54 \pm 0.093^{\mathrm{a}}$ & $8.00 \pm 0.177^{\mathrm{ab}}$ \\
\hline & Overall mean $\pm \mathrm{SE}$ & $7.81 \pm 0.222^{\mathrm{a}}$ & $7.77 \pm 0.205^{\mathrm{a}}$ & $7.63 \pm 0.262^{\mathrm{a}}$ & $7.54 \pm 0.218^{\mathrm{a}}$ \\
\hline \multirow{4}{*}{ Triglycerides, mg/dl } & Pre-parturition & $82.257 \pm 3.450^{b}$ & $93.440 \pm 1.877^{\mathrm{ab}}$ & $94.339 \pm 1.495^{\mathrm{ab}}$ & $102.109 \pm 5.428^{\mathrm{a}}$ \\
\hline & At lambing & $89.555 \pm 9.147^{\mathrm{b}}$ & $103.516 \pm 1.284^{\mathrm{a}}$ & $98.139 \pm 4.202^{\mathrm{ab}}$ & $91.247 \pm 2.534^{\mathrm{b}}$ \\
\hline & At weaning & $97.337 \pm 10.076^{\mathrm{b}}$ & $107.475 \pm 3.017^{\mathrm{ab}}$ & $105.737 \pm 2.056^{\mathrm{ab}}$ & $112.718 \pm 2.386^{\mathrm{a}}$ \\
\hline & Overall mean $\pm \mathrm{SE}$ & $89.716 \pm 4.652^{b}$ & $101.477 \pm 1.636^{\mathrm{a}}$ & $99.405 \pm 1.806^{\mathrm{a}}$ & $102.025 \pm 2.634^{\mathrm{a}}$ \\
\hline \multirow{4}{*}{ Total cholesterol, mg/dl } & Pre-parturition & $97.348 \pm 4.083^{\mathrm{c}}$ & $110.596 \pm 2.221^{b}$ & $111.645 \pm 1.769^{b}$ & $120.841 \pm 6.424^{\mathrm{a}}$ \\
\hline & At lambing & $118.052 \pm 3.912^{\mathrm{ab}}$ & $122.504 \pm 1.519^{\mathrm{a}}$ & $116.140 \pm 4.973^{\mathrm{ab}}$ & $107.986 \pm 2.999^{\mathrm{b}}$ \\
\hline & At weaning & $126.433 \pm 3.417^{\mathrm{a}}$ & $126.854 \pm 3.430^{\mathrm{a}}$ & $125.134 \pm 2.433^{\mathrm{a}}$ & $133.395 \pm 2.825^{\mathrm{a}}$ \\
\hline & Overall mean $\pm \mathrm{SE}$ & $113.944 \pm 3.107^{\mathrm{b}}$ & $119.982 \pm 1.894^{\mathrm{ab}}$ & $117.640 \pm 2.138^{\mathrm{ab}}$ & $120.741 \pm 3.117^{\mathrm{a}}$ \\
\hline \multicolumn{6}{|c|}{ Glucose concentration } \\
\hline \multirow{4}{*}{ Glucose mg/dl } & Pre-parturition & $44.769 \pm 0.438^{\mathrm{a}}$ & $44.189 \pm 2.219^{\mathrm{a}}$ & $34.529 \pm 1.304^{\mathrm{b}}$ & $37.783 \pm 0.586^{\mathrm{b}}$ \\
\hline & At lambing & $67.331 \pm 4.458^{\mathrm{b}}$ & $60.280 \pm 0.294^{\mathrm{c}}$ & $88.723 \pm 0.985^{\mathrm{a}}$ & $64.897 \pm 2.995^{\mathrm{bc}}$ \\
\hline & At weaning & $50.058 \pm 1.303^{\mathrm{c}}$ & $74.60 \pm 0.73^{\mathrm{b}}$ & $80.810 \pm 1.38^{\mathrm{a}}$ & $72.419 \pm 0.508^{\mathrm{b}}$ \\
\hline & Overall mean $\pm \mathrm{SE}$ & $54.053 \pm 2.333^{\mathrm{c}}$ & $59.689 \pm 2.426^{\mathrm{b}}$ & $68.021 \pm 4.487^{\mathrm{a}}$ & $58.366 \pm 2.934^{\text {bc }}$ \\
\hline
\end{tabular}

$a, b$ and $c$, Means within the same row with different superscript are significantly differ $(\mathbf{P}<0.05)$.

In accordance with the present results, ElBassiony (2013) and Shaker (2014) reported that feeding Shami goats and Barki sheep with salt tolerant plants resulted in increasing the activity of AST and ALT. This rise of ALT and AST activities might be attributed to the high tannins (Tripathy et al., 1984), oxalates (Mclntosh, 1972), alkaloids (Craig et al., 1991) and salt (Radostits et al., 1994) in such salt tolerant plants.

In spite of the observed variation in the present study in liver enzymes, all the activities values either for treated or control groups were within the normal physiological reference ranges. It could be concluded that feeding tanniniferous plants did not exert any stress on liver of ewes under any physiological status.

With reference to kidney functions, Urea and creatinine are the two chief nitrogenous composites excreted by kidney. Thus, any change of their concentration would reflect impaired glomerular filtration and/or inefficiency of renal tubules (Kaneko et al. 2008). In the present results, feeding tanniniferous plants shrubs resulted in significant decrease in Urea-N level. The present results come in the same direction with those obtained by Pearce et al. (2008) and Shaker et al. (2014) who reported that Barki sheep that fed a mixture of salt tolerant plants (STP) showed lower values of Urea-N when compared with those fed BH. It worth notice that these plants in spite of having comparable level of $\mathrm{CP}$ with that of $\mathrm{BH}$, but have lower content of EE and NFE while have higher percentages of ash (Table 1). This might mean that the utilization of this protein is low to exert stress on kidney function.

Creatinine level increased obviously only $(\mathrm{P}<0.05)$ in plasma of ewes fed Cassava plant in comparison to other groups. Also creatinine increased from pre- to post-partum stage in all groups. However, in all cases creatinine levels stayed within normal range without any sign for kidney exhaustion. However, Melladoa et al., (2006) and Shaker et al. (2014) reported that feeding STP mixture significantly decreased creatinine levels. On the other hand, Badawy et al. (2002) and Shaker et al. (2008) found that animals fed 
fresh Atriplex or Acacia had higher creatinine levels than those fed BH. EL-Saadany et al. (2016) recorded an increase in creatinine concentration in Barki ewes fed tanniniferous plants shrubs (Acacia, Atriplex and Cassava) and attributed this increase to the antinutritional factors and/or high salt content in these plants.

E. Total antioxidant capacity (TAC):

Feeding tanniniferous plants shrubs did not affect significantly the level of TAC Table (7). The level of
TAC increased from pre- to post-partum only in plasma of ewes fed BH and Acacia. In contrast, Amer et al. (2014) recorded decreased level of TAC in pregnant ewes that fed traditional or untraditional (tanniniferous plants) diets. Also, Nawito et al. (2016) noted that TAC level in blood of local sheep grazing salt tolerant plants in South Sinai was lower than control group, and concluded that, the nutritional level of the diets had very little effect on blood oxidant and antioxidant status.

Table 7. Impact of feeding tanniniferous plants shrubs on liver and kidney functions and total antioxidant capacity (TAC) of ewes in different physiological periods. (Means $\pm \mathrm{SE}$ )

\begin{tabular}{|c|c|c|c|c|c|}
\hline \multirow{2}{*}{ Item } & \multirow{2}{*}{ Sampling time } & \multicolumn{4}{|c|}{ Experimental group } \\
\hline & & Hay & Acacia & Atriplex & Cassava \\
\hline \multicolumn{6}{|c|}{ Liver functions } \\
\hline \multirow{4}{*}{ AST, iu/l } & Pre-parturition & $74.67 \pm 7.745^{\mathrm{b}}$ & $137.83 \pm 21.416^{\mathrm{a}}$ & $114.83 \pm 16.835^{\mathrm{a}}$ & $84.00 \pm 10.415^{b}$ \\
\hline & At lambing & $71.00 \pm 7.933^{\mathrm{ab}}$ & $66.33 \pm 6.339^{\mathrm{ab}}$ & $91.83 \pm 3.439^{\mathrm{a}}$ & $59.00 \pm 5.342^{\mathrm{b}}$ \\
\hline & At weaning & $37.25 \pm 2.007^{\mathrm{a}}$ & $33.00 \pm 0.856^{\mathrm{a}}$ & $37.67 \pm 1.483^{\mathrm{a}}$ & $43.58 \pm 4.623^{\mathrm{a}}$ \\
\hline & Overall mean $\pm \mathrm{SE}$ & $60.97 \pm 5.397^{\mathrm{b}}$ & $79.06 \pm 12.707^{\mathrm{a}}$ & $81.44 \pm 9.524^{\mathrm{a}}$ & $62.20 \pm 5.643^{b}$ \\
\hline \multirow{4}{*}{$\mathrm{ALT}, \mathrm{iu} / \mathrm{l}$} & Pre-parturition & $64.17 \pm 7.167^{\mathrm{a}}$ & $71.17 \pm 3.953^{\mathrm{a}}$ & $67.83 \pm 5.735^{\mathrm{a}}$ & $58.17 \pm 8.089^{\mathrm{a}}$ \\
\hline & At lambing & $56.00 \pm 8.058^{\mathrm{a}}$ & $58.83 \pm 2.822^{\mathrm{a}}$ & $54.50 \pm 5.130^{\mathrm{a}}$ & $45.67 \pm 10.604^{\mathrm{a}}$ \\
\hline & At weaning & $26.67 \pm 1.520^{\mathrm{a}}$ & $22.17 \pm 1.070^{\mathrm{a}}$ & $29.46 \pm 2.082^{\mathrm{a}}$ & $37.79 \pm 6.088^{\mathrm{a}}$ \\
\hline & Overall mean $\pm \mathrm{SE}$ & $48.95 \pm 5.184^{\mathrm{a}}$ & $50.72 \pm 5.282^{\mathrm{a}}$ & $50.60 \pm 4.595^{\mathrm{a}}$ & $47.21 \pm 5.021^{\mathrm{a}}$ \\
\hline \multirow{4}{*}{ ALK-P, iu/l } & Pre-parturition & $0.445 \pm 0.018^{\mathrm{a}}$ & $0.432 \pm 0.034^{\mathrm{a}}$ & $0.421 \pm 0.023^{\mathrm{a}}$ & $0.439 \pm 0.016^{\mathrm{a}}$ \\
\hline & At lambing & $0.382 \pm 0.027^{\mathrm{a}}$ & $0.406 \pm 0.017^{\mathrm{a}}$ & $0.377 \pm 0.019^{\mathrm{a}}$ & $0.372 \pm 0.022^{\mathrm{a}}$ \\
\hline & At weaning & $0.475 \pm 0.009^{\mathrm{ab}}$ & $0.443 \pm 0.009^{b}$ & $0.495 \pm 0.008^{\mathrm{a}}$ & $0.471 \pm 0.007^{\mathrm{ab}}$ \\
\hline & Overall mean \pm SE & $0.434 \pm 0.013^{\mathrm{a}}$ & $0.427 \pm 0.013^{\mathrm{a}}$ & $0.431 \pm 0.013^{\mathrm{a}}$ & $0.427 \pm 0.012^{\mathrm{a}}$ \\
\hline \multicolumn{6}{|c|}{ Kidney functions } \\
\hline \multirow{4}{*}{ Urea-N, mg/dl } & Pre-parturition & $50.41 \pm 1.504^{\mathrm{a}}$ & $36.06 \pm 3.730^{c}$ & $44.14 \pm 1.287^{\mathrm{b}}$ & $46.44 \pm 2.101^{\mathrm{ab}}$ \\
\hline & At lambing & $56.85 \pm 3.430^{\mathrm{a}}$ & $61.28 \pm 1.481^{\mathrm{a}}$ & $59.12 \pm 2.197^{\mathrm{a}}$ & $55.87 \pm 2.126^{\mathrm{a}}$ \\
\hline & At weaning & $86.51 \pm 0.927^{\mathrm{a}}$ & $81.13 \pm 1.955^{\mathrm{a}}$ & $73.99 \pm 1.073^{\mathrm{b}}$ & $71.48 \pm 0.874^{\mathrm{b}}$ \\
\hline & Overall mean $\pm \mathrm{SE}$ & $64.59 \pm 3.173^{\mathrm{a}}$ & $59.49 \pm 3.713^{\mathrm{b}}$ & $59.08 \pm 2.431^{\mathrm{b}}$ & $57.93 \pm 2.163^{\mathrm{b}}$ \\
\hline \multirow{4}{*}{ Creatinine, $\mathrm{mg} / \mathrm{dl}$} & Pre-parturition & $0.563 \pm 0.024^{\mathrm{c}}$ & $0.641 \pm 0.013^{b}$ & $0.648 \pm 0.011^{\mathrm{ab}}$ & $0.700 \pm 0.037^{\mathrm{a}}$ \\
\hline & At lambing & $0.701 \pm 0.016^{\mathrm{ab}}$ & $0.722 \pm 0.007^{\mathrm{a}}$ & $0.699 \pm 0.030^{\mathrm{ab}}$ & $0.656 \pm 0.013^{\mathrm{b}}$ \\
\hline & At weaning & $0.730 \pm 0.020^{\mathrm{a}}$ & $0.735 \pm 0.019^{\mathrm{a}}$ & $0.727 \pm 0.014^{\mathrm{a}}$ & $0.772 \pm 0.016^{\mathrm{a}}$ \\
\hline & Overall mean $\pm \mathrm{SE}$ & $0.665 \pm 0.018^{\mathrm{b}}$ & $0.699 \pm 0.011^{\mathrm{ab}}$ & $0.691 \pm 0.013^{\mathrm{ab}}$ & $0.709 \pm 0.016^{\mathrm{a}}$ \\
\hline \multirow{5}{*}{$\mathrm{TAC} \mathrm{mM} / \mathrm{L}$} & & Total antioxida & capacity & & \\
\hline & Pre-parturition & $0.058 \pm 0.028^{\mathrm{b}}$ & $0.055 \pm 0.018^{\mathrm{b}}$ & $0.206 \pm 0.049^{\mathrm{a}}$ & $0.214 \pm 0.043^{\mathrm{a}}$ \\
\hline & At lambing & $0.268 \pm 0.049^{\mathrm{a}}$ & $0.259 \pm 0.051^{\mathrm{a}}$ & $0.300 \pm 0.045^{\mathrm{a}}$ & $0.202 \pm 0.075^{\mathrm{a}}$ \\
\hline & At weaning & $0.261 \pm 0.057^{\mathrm{a}}$ & $0.236 \pm 0.054^{\mathrm{a}}$ & $0.177 \pm 0.051^{\mathrm{a}}$ & $0.236 \pm 0.040^{\mathrm{a}}$ \\
\hline & Overall mean $\pm \mathrm{SE}$ & $0.196 \pm 0.041^{\mathrm{a}}$ & $0.183 \pm 0.039^{\mathrm{a}}$ & $0.228 \pm 0.031^{\mathrm{a}}$ & $0.217 \pm 0.028^{\mathrm{a}}$ \\
\hline
\end{tabular}

$\overline{a, b}$ and $c$, Means within the same row with different superscript are significantly differ $(\mathrm{P}<0.05)$.

\section{CONCLUSION}

Using tanniniferous plants shrubs, Acacia nilotica, Atriplex nummularia and Cassava Manihot esculenta as alternatives to Berseem Hay did not affect body weights of Barki ewes and their lambs, while improved the milk productivity. In addition, feeding these plants did not cause any negative effects on blood parameters of the experimental animals. Moreover, both kidney and liver functions were not largely affected.

\section{REFERENCES}

A.O.A.C.(1984). Association of official analytical chemistry. Official Methods of Analysis. $\left(14 \mathrm{t}^{\mathrm{h}}\right)$ Edit. Washington; D.C.; USA.

A.O.A.C. (1995). Association of official analytical chemistry. Official Methods of Analysis. $\left(16^{\text {th }}\right)$ Edit. Washington; D.C.; USA.
Abdel-Bary, H. T. M. (1990). Blood plasma protein levels during different physiological stages of fattailed ewes. Al-Azhar J. Agric. Res., 12: 113128.

Abdelhameed, Afaf A. E., Shawket, M. Safinaz and Kewan, K. Z. (2006). Physiological studies of feeding halophytes on some blood parameters and body fat of camels under desert condition. $4^{\text {th }}$ Sci. Conf., 29-30 July, Giza, Egypt, pp. 157-180.

Abu-Zanat, M. M. W. and Tabbaa, M. J. (2006). Effect of feeding Atriplex browse to lactating ewes on milk yield and growth rate of their lambs. J. Small Rum. Res. pp152-161.

Ahmed, M. A., El-Metoly, H. A., Khir, A. A. and Deraz, T. A. (2013). Effect of feeding she-camel by desert plants on the milk yield, composition and the probiotic yoghurt properties made from the milk. Egypt. J. of Appl. Sci. 28: 352-371. 
Amer, H., Ibrahim, N. H., Donia, G. R., Younis, F. E. and Shaker, Y. M. (2014). Scrutinizing of trace elements and antioxidants enzymes changes in Barki ewes fed salt-tolerant plants under South Sinai condition. J. Amer. Sci., 10(2): 241-249.

Badawy, M.T., Gawish, H. A. and Younis, A. A. (2002). Some physiological responses of growing Barki lambs and Baladi kids fed natural desert shrubs. International Symposium on Optimum Resources Utilization in Salt-Affect Ecosystems in Arid and Semi-arid Regions. 8-11 April, Cairo, Egypt, pp. 496-503.

Barnett, A. S. and Abdel-Tawab, G. (1957). A rapid method for determination of lactose in milk and cheese. J. sci. Food Agric., 8; 437.

Ben Salem, H., Nefzaoui, A. and Ben Salem, L. (2002). Supplementation of Acacia cyanophylla Lindi. Foliage-based diets with barley or shrubs from arid areas (Opuntiaficus-indica f. inermis and Atriplex nummularia L.) on growth and digestibility in lambs. Anim. Feed Sci. Tech. 96:15-30.

Ben Salem, H., Nefzaoui, A., Makkar, H. P. S., Hochlef, H., Ben Salem, I. and Ben Salem, L. (2005). Effect of early experience and adaptation period on voluntary intake, digestion, and growth in Barbarine lambs given tannin-containing (Acacia cyanophylla Lindl, foliage) or tannin-free (oaten hay) diets. Anim. Feed Sci. Tech. 122: 59-77.

Craig, A. M., Pearson, E. G., Meyer, C. and Schmitz, J. A. (1991). Serum level enzyme and histopathologic changes in calves with chronic and chronoic- delayed Senecio jacobaea toxicosis. Amer. J. Vet. Res., 52 (12): 1969-1678.

Degan, A. A., Blankc, A., Beker, K., Kam, M., Benjamin, R. W. and Makkar, H. P. S. (1997). The nutritive value of Acacia saligna and Acacia salcina for goats and sheep. J. Anim. Sci., 64: 253-259.

Duncan, D. B. (1955). Multiple Range's and Multiple Ftest. Biometrics, 11: 1-42.Ver. 8.0 Edit.. SAS Inst., Inc., Cary, NC.

El-Bassiony, M. F. (2013). Productive and reproductive responses of growing Shami goat kids to prolonged saline conditions in South Sinai. Ph.D. Thesis, Fac. Agric., Cairo Univ., Egypt.

El-Hawy, A. S. (2013). Reproductive efficiency of Shami goats in salt affected lands in South Sinai. Ph.D. Thesis, Fac. Agric., Ain Shams Univ., Egypt.

EL-Saadany, S. A., H. EL-Gohary, E. S., Omar, H. H., ELwakeel, EL .A. and EL-sayed, I. A. (2016). Impact of feeding some forage shrubs on milk, blood parameters of ewes and growth performance of their offspring's in north coast. J. Anim. and Poult. Prod., Mans. Univ., vol. 7 (1): 47-57.

El-Shaer, H. M. and Gihad, E. A. (1994). Halophytes as animal feeds in Egyptian deserts. Proc. of the Inter. Workshop on "Halophytes for rehabilitation of saline wastelands and a resource for livestock: Problems and prospects". Nov. 2227, Nairobi, Kenya. pp. 281-284.
El-Shaer, H. M., Kandil, H. M., Abou El-Nasr , H. M. and Khamis, H. S. (1997). Features and constrains of animal resources development in Shalateen-Halaib region. Egypt. J. Nutr. and feeds (Special Issue): 121 - 128.

Fasae, O. A., Amos, A. O., Owodunni, A. and Yusuf, A. O. (2015). Performance, haematological parameters and faecal egg count of semiintensively managed West African dwarf sheep to varying levels of Cassava leaves and peels supplementation. Pertanika J. of Trop. Agric. Sci.; 38(1): 71-81.

Haenlein, G. F. W. (2001). The nutritional value of sheep milk. Inter. J. Anim. Sci., 16-253.

Hassan, N. I. and Abd El-Aziz, H. M. (1979). Effect of barley supplementation on the nutritive value of saltbush (Atriplex nummularia). World Rev. Anim. Prod., 15: 47.57.

Hassan, A. A., Salma, H. Abou Hafsa, Yacout, M. H., Khalel, M. S., Ibrahim M. A. R. and Dorina Mocuta. (2015). Effect of feeding some forage shrubs on Goats performance and rumen fermentation in dry season. Egypt. J. of Sh. and G. Sci., Proc. Book of the $5^{\text {th }}$ Intr. Sci. Confer. on Small Rum. Prod., Sharm El Sheikh-Egypt, pp. 21-36.

Ibrahim, S. M. N. (2001). Alteration of blood components and histopathological changes in goats exposed to some range plants growing in the Egyptian desert. Ph. D. Thesis. Fac. of Vet. Mid. Clini. Path., Cairo Univ.

Ling (1963). A Text Book of Dairy Chemistry. $3^{\text {rd }}$ Edit., Vol. 2 Chapman and Hall London UK.

Kaneko, J. J., Harvey, J. W. and Bruss, M. L. (2008). Clinical Biochemistry of Domestic Animals. $6^{\text {th }}$ Edit. Elsevier/Academic Press, Amsterdam.

Mclntosh, G. H. (1972). Chronic oxalate poisoning in sheep. Aust. Vet., 48: 535-545.

Melladoa, M., Olivaresa, L., Rodrigueza, A. and Melladoa, J. (2006). Relation among Blood Profiles and Goat Diets on Rangeland. J. of Appli. Anim. Res., Vol. (30): 1, 93-98.

Menzies, P. I. and Ramanoon, S. (2001). Mastitis of sheep and goats. Vet. Cline. North Amer., Food Anim. Pract. 17(2): 333-358.

Mohammady, M. I., Hammam, A. H. and Ibrahim, N. H. (2014). Returns and Economical Efficiency of Barki Sheep Fe don Salt Tolerant Plants in Sinai, Egypt. J. of Amer. Sci. 10 (4):134-139.

Nawito, M. F., Abd El Hameed, A. R., Sosa, A. S. A. and Mahmoud, Gh. K. (2016). Impact of pregnancy and nutrition on oxidant/antioxidant balance in sheep and goats reared in South Sinai, Egypt. Vet. World pp. 281-284.

NRC. (1985). National Research Council. Nutrient Requirements of Sheep, $6^{\text {th }}$ Edit., National research council, National academy press, Washington, D.C. USA. 
Pearce, K. L., Pethick, D.W. and Masters, D.G. (2008). The effect of ingesting a saltbush and barley ration on the carcass and eating quality of sheep meat. Anim. Sci. 2(3): 479-490.

Pengov, A. (2001). The role of CNS and associated SCC in the ovine mammary gland. J. Dairy Sci. 84(3): 572-574.

Radostits, O. M. Blood, D. C. and Gat, C. C. (1994). "Veterinary medicine". $8^{\text {th }}$ Edit. Bailliere Tindall, London, England.

SAS., (2004). Statistical Analysis System, SAS User's Guide Statistics, SAS Institute Inc., Edit. Cary NC, USA.

Shaker, Y. M., Abou El-Ezz, S. S. and Hashem, A. L.(2008). Physiological performance of Barki male lambs fed halophytes under semi-arid conditions. J. Agric. Sci. Mans. Univ. 33(9): 6393-6408.

Shaker, Y. M., Ibrahim, N. H., Younis, F. E. and El Shaer, H. M. (2014). Effect of Feeding Some Salt Tolerant Fodder Shrubs Mixture on Physiological Performance of Shami Goats in Southern Sinai, Egypt. J. of Amer. Sci.;10 (2s): 66-76.

Shaker, Y. M. (2014). Live Body Weight Changes and Physiological performance of Barki Sheep fed salt tolerant fodder crops under the arid conditions of Southern Sinai, Egypt. J. Amer. Sci. 10(2s): 78-88.

Shawket, S. M. and Ibrahem, A. H. (2012). Proceedings of the $3^{\text {rd }}$ Conference of the International Society of Camelid Research and Development (ISOCARD), $29^{\text {th }}$ Jan. $-1^{\text {st }}$ Feb., Muscat, Sultanate of Oman.
Shawket, S. M., Ahmed, M. H. and Ibrahim, M. A. (2010). Impact of feeding Atriplex halimus and Acacia saligna with different sources of energy on lamb's performance. Egypt. J. of Sh. and G. Sci., Vol. 5 (1): 191-208.

Shetaewi, M. M., Abdel-Samee, A. M. and Bakr, E. A. (2001). Reproductive Performance and Milk Production of Damascus Goats Fed Acacia Shrubs or Berseem Clover Hay in North Sinai, Egypt. Trop. Anim. Health \& Prod. 33(1): 67-79.

Squires, V. R. and Ayoub, A.T. (1994). Halophytes as resource for livestock and for rehabilitation of degraded lands. Proc. of the Inter. Workshop on "Halophytes for reclamation of saline wasteland and as a resource for livestock: Problems and prospects", Kluwer Academic Publishers, London.

Tripathy, K. C., Sahu, B. K., Panda, N. C. and Nayak, B. C. (1984). Toxicity of tannin acid in goats. Indian J. Anim. Sci., 54- (11): 1091-1093.

Vongsamphanh, P. and Wanapat, M. (2004). Comparison of Cassava hay yield and chemical composition of local and introduced varieties and effects of levels of Cassava hay supplementation in native beef cattle fed on rice straw. Livestock Res. for Rural Develop., 16(8): 2004.

Warren, B. E., Bunny, C. I. and Bryant, E. R. (1990). A preliminary examination of the nutritive value of four saltbush (Atriplex) species. Proc. Austr. Soc. Anim. Prod. 18: 424-427.

Wiggans, G. R. and Shook, G. E. (1987). A lactation measure of somatic cell count. J. of dairy Sci., 70 (12), 2666-2672.

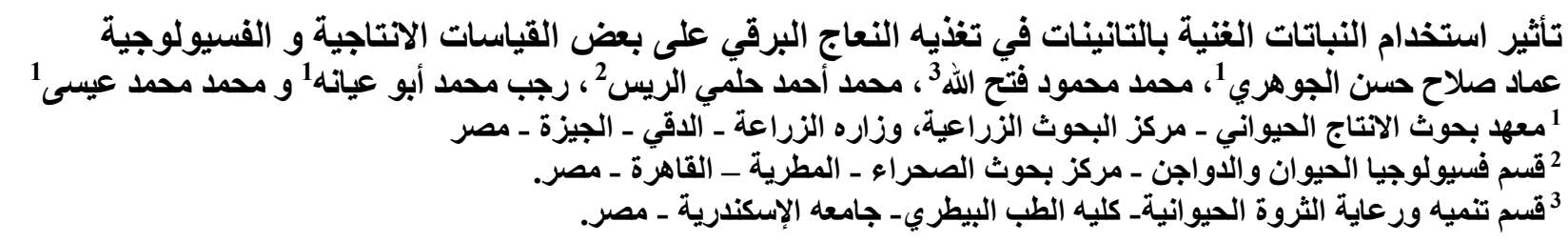

استخدم في هذا البحث أربعون نعجة برقى ناضجة في أواخر فترة الحمل ، تتراوح أعمار ها بين 3-4 سنه وبمتوسط

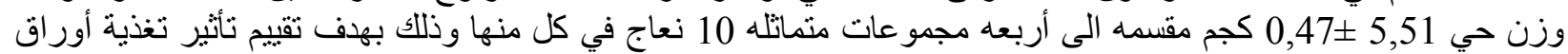

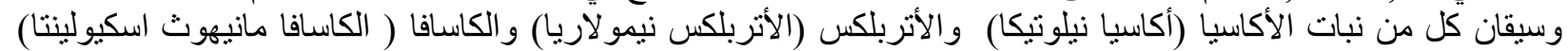

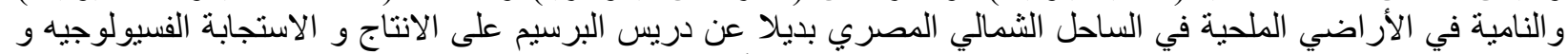
بعض مكونات اللبن والدم الحيوية. غذيت فيت النعاج في المجمو عة الأولى (مج1) على عليقة المقارنة (دريس) و التى تكونت من النى

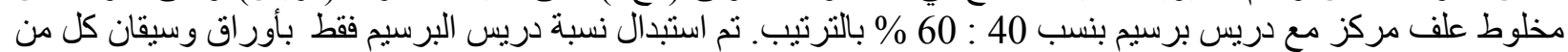

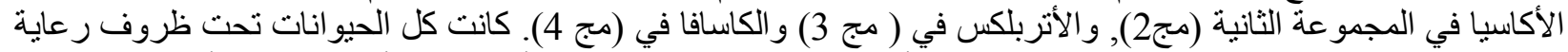

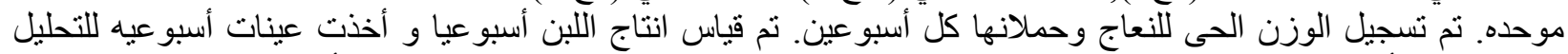

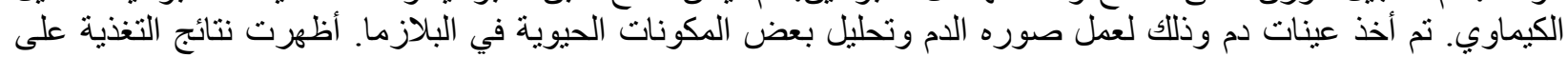
النباتات الثجيرية والغنية بالتانينات الى عدم وجود فروق معنوية في وزن الجسم للنعاج البرقي وحملانها بينما تحسنت التتاجيه

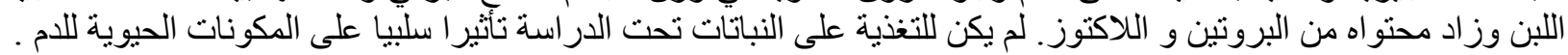

